\title{
Using Hardware Classification to Improve PC-Based OpenFlow Switching
}

\author{
Voravit Tanyingyong, Markus Hidell, Peter Sjödin \\ School of Information and Communication Technology \\ KTH Royal Institute of Technology \\ Kista, Sweden \\ Email: \{voravit, mahidell, psj\}@kth.se
}

\begin{abstract}
OpenFlow is a promising technology that offers a flexible flow-based forwarding scheme based on multiple fields in the packet headers of different protocol layers. It provides a feasibility to adopt new network protocols and innovations thanks to a more flexible forwarding compared to traditional IP forwarding. In this paper, we propose an architectural design to improve lookup performance of PC-based OpenFlow switching in Linux using a standard commodity network interface card based on the Intel 82599 Gigabit Ethernet controller. We introduce a fast data path based on caching of flow table entries in on-board classification hardware on the NIC. We describe our design, and we present an experimental evaluation of its performance. Our results show that the throughput of our fast path gives over 40 percent higher throughput compared to the regular softwarebased OpenFlow switching.
\end{abstract}

\section{INTRODUCTION}

In recent years, there has been a strong trend in the networking community to address network ossification using a clean slate approach. Network virtualization plays a major role in this effort thanks to its possibilities to support innovation through disruptive technologies [1]. Through the means of virtualization, each infrastructure node can be sliced to allow multiple network users to reserve resources and simultaneously share the infrastructure. Furthermore, each slice is programmable allowing network users to control its properties and behavior. Large-scale projects such as FEDERICA [2], GENI [3], and AKARI [4] are good examples of this trend.

Taking a step further in this direction, Software-Defined Network (SDN) comes forth as a new way to repartition networks [5]. In SDN, there is a clear separation between the data plane and control plane. The data plane is a packetforwarding path (i.e., switches, routers, etc.) whereas the control plane is a centralized network-wide operating system known as a network $O S$. The network OS has a global view of the network state and is hosting network features such as routings, access control, etc. It controls the data plane through an open vendor-independent API (such as OpenFlow [6]).

OpenFlow [6] is a novel technology that enables flow tables in switches and routers to be programmed via a standardized interface: the OpenFlow protocol. OpenFlow switching is flow-based-a forwarding paradigm based on multiple fields of packet headers at different protocol layers-which is more flexible compared to traditional IP forwarding. OpenFlow is a promising technology that facilitates a clear separation between the data plane and control plane in the SDN architecture.

A software-based OpenFlow reference implementation, suitable for further development, is publicly available [7]. Although it is general and flexible, it inherits lookup performance penalties that come with software processing. OpenFlow switching implies that more information needs to be taken into account in the packet lookup compared to regular IP forwarding. In addition, OpenFlow's switching flexibility, gained by allowing wildcard lookups, results in reduced performance when implemented in software since such implementations often come down to doing linear searches in the lookup tables. In fact, such effects on the performance can be seen in the OpenFlow switching performance experiments done in [8]. To enhance software-based OpenFlow lookups, we suggest an extension to accelerate the performance.

In this paper, we propose an open architecture based on the Intel 8259910 Gigabit Ethernet (GbE) controller [9] to improve the lookup performance of the OpenFlow Linux kernel module implementation. We do this by using a hardware classification feature available on the network interface card (NIC). This idea to offload packet processing from the host CPU level to on-board NIC hardware is similar to earlier work [10], [11], but we use a different approach based on a regular commodity NIC rather than specialized NICs with FPGAs or network processors (NPs). A commodity NIC might not be as flexible, but it has a more competitive price and better availability. Moreover, doing modifications based on specialized NICs can be difficult since it usually requires complex low-level programming or specialized software packages whereas commodity NIC's driver uses standard programming languages such as $\mathrm{C}$ or $\mathrm{C}++$. Furthermore, the Intel NIC offers higher line speed compared to the related work $(10 \mathrm{GbE}$ rather than $1 \mathrm{GbE})$. This paper is a continuation of our earlier work [12], in which we did some preliminary investigations with respect to the potential performance gains. The results were encouraging, so therefore we explore a general solution for offloading OpenFlow lookups. Our proposed solution is based on caching flow table entries in on-board classification hardware on the NIC.

The rest of this paper is organized as follows; Section II describes our architectural design. It also includes details of the key technologies we employ in the architecture. Section 
III covers experiments and evaluations. Finally, we conclude our work in Section IV.

\section{ARchitectural Design}

To improve OpenFlow lookup performance, it is important to have a clear understanding of the OpenFlow concept in general as well as of the implementation, in particular the packet forwarding procedure. The OpenFlow reference system [7] comes with two forwarding implementations: a user-space implementation and a Linux kernel module implementation. We focus on the kernel module implementation since it offers better performance compared to the user space implementation.

\section{A. OpenFlow Technology and Implementation}

The OpenFlow technology is based on an Ethernet switch and comprises of three main components, which are

- a flow table that contains entries of flows and associated actions the switch should perform on the recieved flows.

- a secure channel that provides a secure communication path between the switch and a remote process (called controller).

- an OpenFlow protocol implementation that provides a standard way for a controller to configure and manage the switch.

In OpenFlow, a flow is defined based on layer 2-4 information. Wildcards can be used for any of these fields, which makes OpenFlow very flexible for flow classification and lookup. The lookup mechanism in OpenFlow is straight forward. Once the received packet arrives to the OpenFlow switch, it will be matched against entries in the flow table. An action will be performed as defined in the matching flow table entry. This action can be to drop or to forward the packet (including forwarding to the controller).

The OpenFlow kernel module is the OpenFlow implementation in Linux kernel. It comes with two different lookup tables; one hash-based lookup table and one linear lookup table. In general, a hash-based lookup table gives better performance compared to linear lookups. However, a hash table is not designed for lookups involving wildcard fields, which is one of the key properties to provide flexibility in OpenFlow. Therefore, the OpenFlow implementation uses a hash-based lookup table for exact match and it uses a linear lookup table to support wildcard lookups. The linear table can match any type of flow entries, but a linear search in a large table is a time-consuming task. The OpenFlow implementation has a default maximum of $64 \mathrm{~K}$ entries for the hash table and 100 entries for linear table.

In the OpenFlow implementation, the lookup is performed in two steps as illustrated in Fig. 1. When a packet arrives, the packet header is hashed and checked against the hash table. If a match is found, then an action is performed as defined in the matching flow entry. If no match is found, a search for a match in the linear table is done and the corresponding action is performed upon a match.

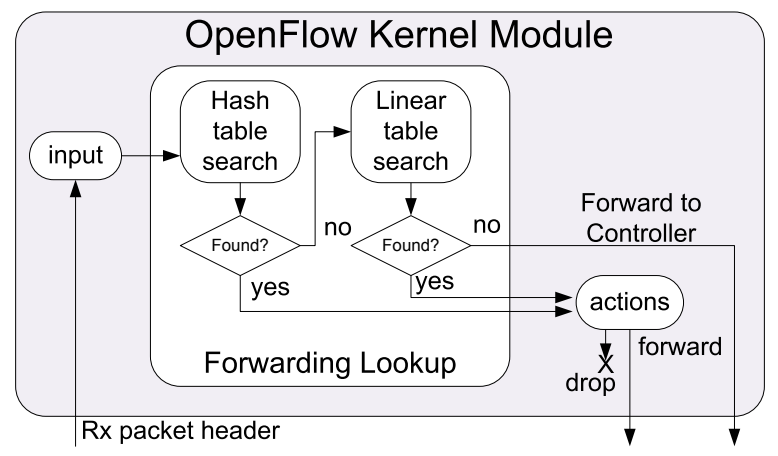

Fig. 1. OpenFlow lookup in Linux kernel module implementation

\section{B. Design Motivation}

We aim at keeping our design open and accessible by using open source software and standard PC hardware components. From the software side, the publicly available OpenFlow kernel module matches well with our purpose. From the hardware side, technology advancement enables today's commodity off-the-shelf hardware components to provide performance improving features that could be compared with specialized hardware.

Processor manufacturers have shifted their design focus from increasing clock speeds to increasing the number of cores. In response to such multi-core architectures, network devices with multiple queues have emerged. Each queue can be mapped to a CPU core via CPU affinity. This helps improving cache behavior, and enables multiple CPU cores to perform packet processing concurrently on a single interface, which leads to an increase of the overall packet processing performance on a standard computer. Accordingly, we consider multi-core processor architectures and multi-queue network interface cards as crucial elements of our system architecture.

Unlike specialized NICs, such as those based on FPGAs or network processors, commodity NICs have no capability to forward the packet by themselves. They depend on packet processing in the CPU. Our target is to offload the CPU as much as possible.

With our design objectives in mind, we propose an enhancement of the OpenFlow lookup process by utilizing classification features supported by the Intel $8259910 \mathrm{GbE}$ controller. This is a very recent chipset, which Intel uses in all new 10 GbE NICs. It is rich in features and well-suited to our needs. The key feature of interest that we employ in our packet lookup enhancement is called Flow Director.

\section{Flow Director}

The Flow Director provides filters that direct received packets, according to their flows, to queues for classification purposes. The filter covers the following fields in the packet headers: VLAN header, Source IP, Destination IP, Source port, Destination port, IPv4 or IPv6 protocol match, L4 protocol match (UDP/TCP/SCTP), and a flexible 2-byte tuple anywhere in the first 64-bytes of the packet header. 


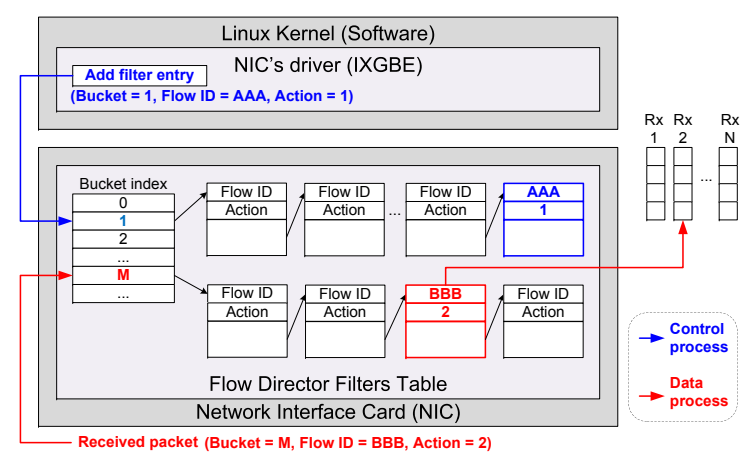

Fig. 2. Control process and Data process of Flow Director

A filter entry can be programmed based on the packet header fields to define a flow and a receive queue that this flow should be directed to. In addition, a mask to provide a range for matching is supported by the Flow Director. This mask is defined globally and then used for all filters. All filter entries are kept in a single filter table, and the Flow Director uses a hash (based on relevant fields) lookup table to create a bucket index. Each bucket is then organized in a variablelength linked list.

The Flow Director offers two types of filtering modes, namely signature filter and perfect match filter. For both filter modes, the matching is done against a flow ID. Signature filters use hash-based flow IDs, which support larger number of filter entries. However, it might result in a false positive since multiple flows could have the same bucket hash and signature (flow ID) values. Perfect match filter guarantees to have no false positive. Therefore, we use perfect match in our architecture. The maximum number of filter entries is $8 \mathrm{~K}$ for perfect match filter and $32 \mathrm{~K}$ for signature filter.

The Flow Director can be divided into two main functional processes; the control process and the data process. The control process is basically about programming filters and monitoring of the filter entries. The data process involves matching received packets against the programmed filters. When a packet arrives to the NIC, the relevant packet header fields are used to compute a flow ID as well as a bucket index for using as keys in the search process. Once the matching filter is found, the programmed action can be performed, which is to direct the packet to the desired receive queue.

Fig. 2 illustrates the control process, in which a new filter with flow ID AAA is added to bucket index 1, and the data process, in which a received packet with flow ID BBB is matched against the entries in the filter table's linked list in bucket index $\mathrm{M}$ and subsequently being directed to receive queue 2.

The Flow Director is originally intended for controlling data streams (or flows) to/from the application layer on the host machine. However, when it comes to what we intend to use it for, i.e. offloading the OpenFlow lookups, it has some limitations. First, the Flow Director does not support the complete set of fields used in OpenFlow switching. These unsupported fields are ingress port, Ethernet source, Ethernet

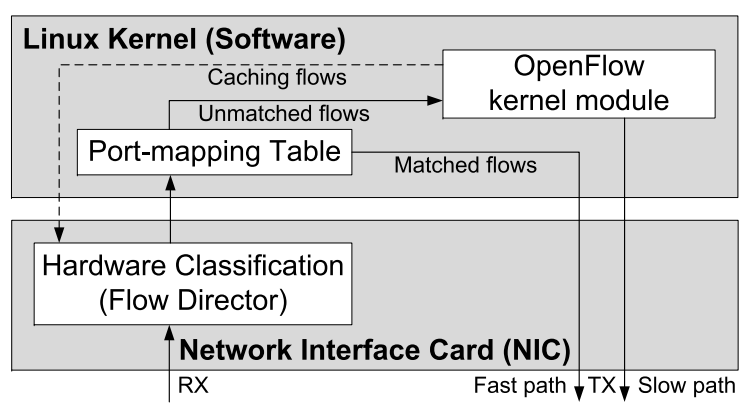

Fig. 3. Architectural Design

TABLE I

PORT-MAPPING TABLE

\begin{tabular}{|c|c||c|c|}
\hline Input port & Input queue & Output port & Output queue \\
\hline Rx IfIndex & Rx queue & Tx IfIndex & Tx queue \\
\hline
\end{tabular}

destination, Ethernet type, IP protocol numbers (all IP), and IP ToS bits. This means that not all lookup entries in OpenFlow can be transparently moved to the Flow Director's filter table. Some entries that contain fields not supported by the Flow Director will still need to be kept in the OpenFlow lookup table. Second, the Flow Director allows only one global mask. This means that it is impossible to move filter entries with different types of wildcards to the Flow Director's filter table. Instead, our approach is based on identifying individual flows matching a wildcard entry, and installs the corresponding specific flow entries in the Flow Director.

\section{System Architecture}

Our goal is to improve the lookup performance of PCbased OpenFlow with the use of NIC hardware classification to offload the CPU as much as possible from the lookup processing. Thus, we propose an architecture based on caching of flows as depicted in Fig. 3.

We introduce a fast path in the lookup process to bypass the OpenFlow kernel module, which we consider as a bottleneck. This is done by caching active flow table entries in the Flow Director. When packets arrive, the Flow Director sorts the incoming packets to queues for matched packets and unmatched packets accordingly and passes them on to the software (the Linux kernel). Then, the actual forwarding decision on which path each received packet will take is made in the port-mapping table. In the port-mapping table (as depicted in Table I), the receive interface and recieve queue are used as a lookup key to quickly find the output interface and output queue for a packet (forwarding along the fast path). A packet belonging to a cached flow will have a match in the port-mapping table and gets forward directly, while a packet that does not belong to a cached flow will have no match and thus takes the slow path through a completely software-based OpenFlow lookup.

The multi-queue feature of the NIC makes it possible to load-share packet processing among multiple CPU cores. 


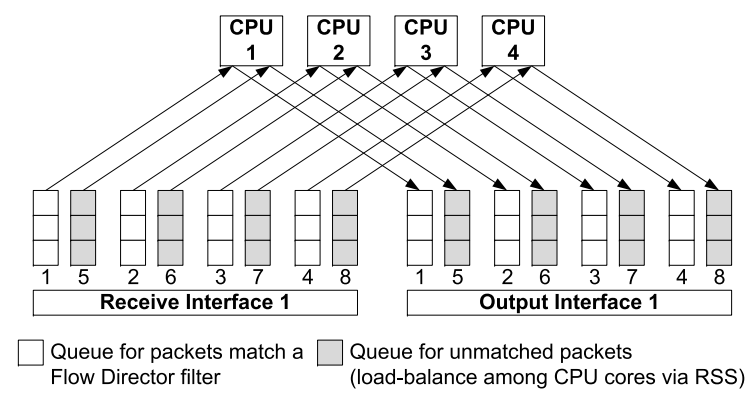

Fig. 4. Queue mappings to CPU cores

\begin{tabular}{|c|c|c|c|c|}
\hline $\begin{array}{c}\text { PC1 } \\
\text { Pktgen sender } \\
\text { (Source) }\end{array}$ & $\begin{array}{c}10 \\
\mathrm{GbE}\end{array}$ & $\begin{array}{c}\text { PC2 } \\
\text { OpenFlow switch } \\
\text { (DUT) }\end{array}$ & $\begin{array}{c}10 \\
\mathrm{GbE} \\
\end{array}$ & $\begin{array}{c}\text { PC3 } \\
\text { Pktgen receiver } \\
\text { (Sink) }\end{array}$ \\
\hline
\end{tabular}

Fig. 5. Experiment Setup

Taking this into consideration, we allocate two receive queues to each CPU core for each receive interface as illustrated in Fig. 4. One queue is used for matched incoming packets and the other for unmatched packets. This method of queue mapping ensures that only one queue is used for directing a matched packet to a specific CPU core, which reduces the CPU contention among receive queues. Furthermore, we take advantage of the Receive-Side Scaling (RSS) feature on the NIC by allocating one unmatched queue per CPU core. These queues are used for distributing the unmatched packets among the CPU cores for load sharing.

In our current implementation, the caching mechanism automatically adds a flow entry to the Flow Director when the first packet of a flow matches an entry in the OpenFlow lookup tables. We will refer to our architecture as hardware classification extension from this point onwards.

\section{EXPERIMENTAL EVALUATION}

\section{A. Experiment setup}

We adopt a standard experiment setup in conformance with RFC 2544 [13] using three PCs to evaluate the forwarding performance of our architecture; $\mathrm{PC} 1$ is a traffic generator (source), PC2 is a Device Under Test (DUT) with OpenFlow kernel module version 0.8.9-rev4, and PC3 is a traffic receiver (sink). Pktgen [14] is used as a packet generator on PC1, and Pktgen with a patch for receiver side traffic analysis [15] is used on PC3. All three PCs have identical hardware; TYAN S7002 Motherboard with Intel Xeon Quad Core 5520, $2.26 \mathrm{GHz}, 3 \mathrm{~GB}$ RAM, one $10 \mathrm{GbE}$ dual-port NIC with Intel $8259910 \mathrm{GbE}$ controller. The operating system we use is Bifrost/Linux [16], which is a Linux distribution optimized for routing. We use Bifrost 6.1 with the Linux net-next 2.6.34-rc2 kernel. The experiment setup is depicted in Fig. 5.

\section{B. Experiment scenario}

To investigate the performance and flexibility aspects of our hardware classification extension, we carry out various experiments as follows:
1) Baseline Performance Test: This is a test to find the baseline performance of hash and linear lookups in standard OpenFlow and compare their performance with port-mapping lookups in the modified version of OpenFlow that incorporates hardware classification extension. We are interested both in the maximum throughput and in how the throughput is affected by the number of entries in the respective tables.

To keep the test simple, we set up our OpenFlow switch to use only one CPU core and two receive queues. Both receive queues are mapped to the $\mathrm{CPU}$ core. One queue is used for matched flows and the other queue is used for unmatched flows. To make a fair comparison, we configure standard OpenFlow so that only one queue is used in hash and linear lookups as well. We carry out a test for each lookup table, namely OpenFlow's linear lookup table, OpenFlow's hash lookup table, and the hardware classification extension's port-mapping lookup table. The forwarding rule is simply to forward packets that match entries in the lookup table. For linear lookup, we use source/destination IP addresses and port numbers for matching (the rest of the fields are wildcards). For hash lookup, we use exact match. For the port-mapping table, we add 5-tuple (source/destination IP addresses, port numbers, and IP protocol) flow entries in the Flow Director filter.

We measure performance for varying numbers of table entries. The performance metric we focus on is the throughput in terms of packet per second (pps). In each test, PC1 sends randomized 64-byte packets from 100 different UDP flows. PC2 (the OpenFlow switch) receives all traffic on one port and forwards them on another port to PC3. All generated flows from PC1 will match entries in the lookup table. We generate traffic corresponding to an overload situation with loads between 1 and 5.5 Mpps, which is the highest load that our sink (PC3) can receive without dropping any packet.

The test result is shown in Table II. The throughput tendency of each lookup table is as expected. With a linear table, the throughput decreases when table size increases. With the hash table and the port-mapping table, the throughput is not affected by the number of entries in the tables.

Moreover, our hardware classification extension improves OpenFlow switching throughput significantly. On average, the increase is $41.50 \%$, or roughly 239,400 pps. As we shall see later, the overall performance increases as we increase the number of CPU cores.

2) Modification Overhead: It is clear from the baseline performance test that the hardware classification extension can significantly improve OpenFlow switching performance. However, it could create an extra overhead for OpenFlow switching when there is no match in the Flow Director. In this situation, there will be a miss in the port-mapping table (the outgoing port will not be found). The overhead is the additional time it takes to perform this lookup in the port-mapping table before entering the standard OpenFlow lookup. This means that the overhead is the difference between the throughput of standard OpenFlow hash lookup and the throughput when doing a hash lookup after a miss in the portmapping table. 
TABLE II

Baseline Performance Test

\begin{tabular}{|c||c|c|c|}
\hline \#Entries & $\begin{array}{c}\text { Linear } \\
\text { (in pps) }\end{array}$ & $\begin{array}{c}\text { Hash } \\
\text { (in pps) }\end{array}$ & $\begin{array}{c}\text { Port-mapping } \\
\text { (in pss) }\end{array}$ \\
\hline 1 & 523275 & - & 813717 \\
100 & 359553 & 580606 & 816446 \\
1100 & 82470 & 577795 & 817455 \\
2100 & 45159 & 576938 & 815952 \\
3100 & 31173 & 576666 & 816497 \\
4100 & 23988 & 576375 & 815637 \\
5100 & 19528 & 575995 & 817427 \\
6100 & 16478 & 576256 & 817897 \\
7100 & 14262 & 575923 & 816292 \\
8100 & 12589 & 575067 & 814788 \\
\hline
\end{tabular}

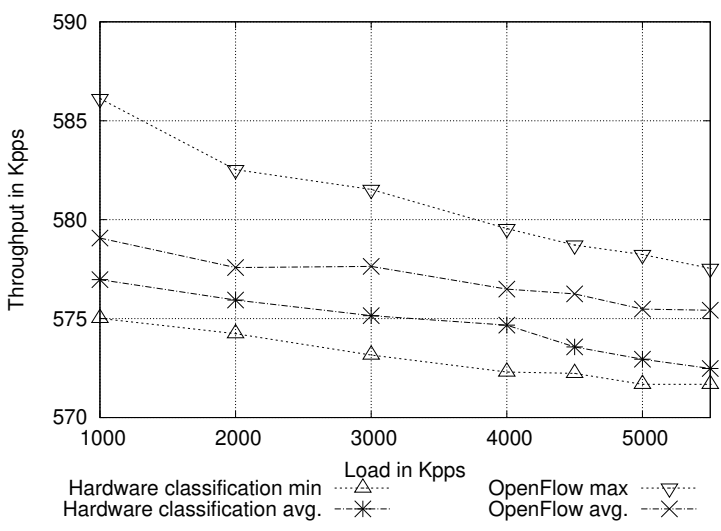

Fig. 6. Overhead of Hardware Classification Extension

To find the overhead incurred by misses in the port-mapping table lookup, we create a scenario where all the matched entries are in hash table and no flows will ever have a match in the Flow Director. All lookups then have to go through portmapping lookup table before finding a match in hash table. To ensure that there will always be no filter entry in the Flow Director, we remove the caching capability from the NIC's driver in this experiment. We use the same setup as in the baseline performance test with one CPU core and two receive queues. The experiment uses the same sets of pre-defined filter entries and load values.

The overhead is only visible when the system is saturated. Thus, we select to plot the average values of when we saturate the system (with loads between $1 \mathrm{Mpps}$ and 5.5 Mpps) as illustrated in Fig. 6. The average overhead is $0.40 \%$ (roughly 2,300 pps). To create the worst case results, we also compare the minimum throughput values of hash lookup in the hardware classification extension to the maximum throughput values of hash lookup from the baseline performance test. The maximum overhead in this comparison is $1.90 \%$ at 1 Mpps load. Considering the gain that we can achieve from the baseline performance test, this overhead is negligible. The gain from having matched flows is much more significant. Hence, we regard this as a sound trade-off.

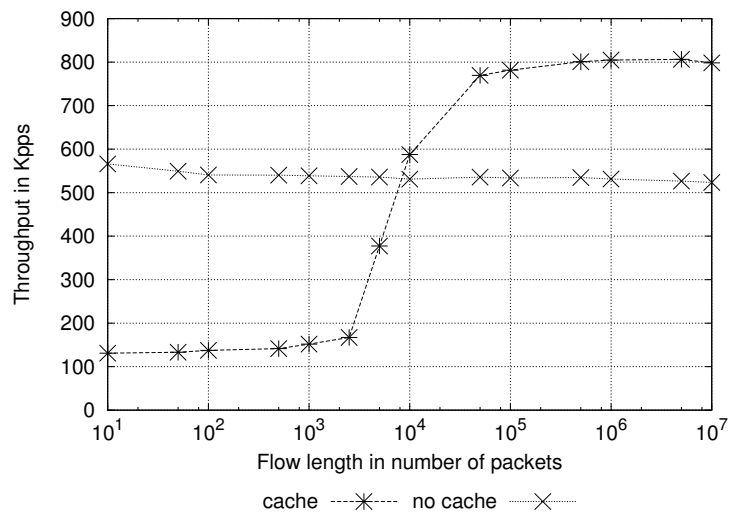

Fig. 7. Influence of Flow Length

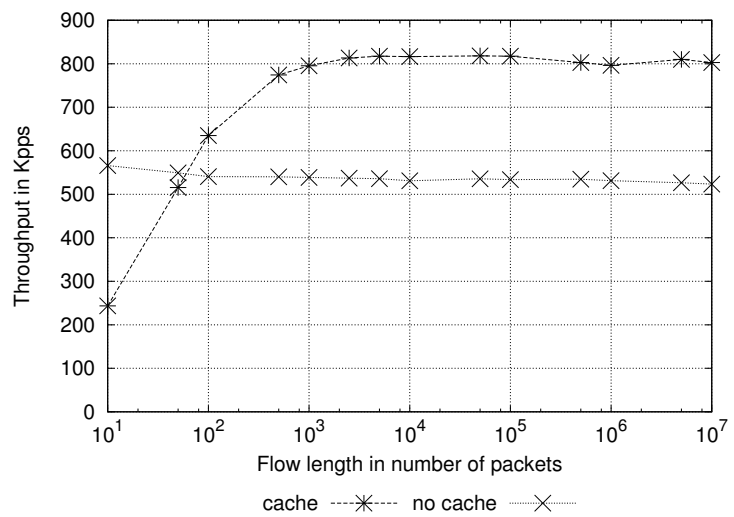

Fig. 8. Influence of Flow Length with randomized flows

3) Flow caching: There is a certain cost associated with caching a flow in the Flow Director for hardware classification. The operation of configuring an entry in the Flow Director takes a few microseconds ( 3.3 on the average). To make it worth caching a flow, this cost needs to be amortized over a certain number of subsequent packets belonging to the same flow. Therefore, we need to investigate how the flow length (the total number of packets in a flow) affects the performance. In particular, we are interested in the flow length needed to make a gain from using flow caching. We therefore compare throughputs of the flow caching with throughputs of the standard OpenFlow for various flow lengths. The purpose is to find the break point where we make a gain using the hardware classification extension. We use the setup with one CPU core and two receive queues similar to the baseline performance test setup. Then, we populate the linear table with one entry that matches all UDP flows. The traffic generator on PC1 sends 64-byte packets of 100 UDP flows in sequential order at full speed, one flow at a time. Each flow is sent with a fixed flow length. For each test round, we increase the flow length. This traffic pattern generates the shortest possible life time for each flow. Sending a large number of minimum-size packets at full speed might not be very realistic but it should give a worst-case scenario for the flow caching scheme. 
The test result in Fig. 7 shows that throughput increases with the flow length, as expected. There is a break point showing that flow caching gives higher throughput than without caching when the flow length is 10000 packets. However, this test is a worst case scenario for flow caching since the different flows come in sequence and therefore the packets of a particular flow come back to back. In a more realistic traffic pattern, packets from different flows will be interleaved so that the cost of configuring a flow entry in the cache has a smaller influence on the overall performance. To verify this, we also run a test where the packets from the different flows are randomly interleaved. This is still a demanding scenario since we send the minimum-size packets at full speed. The test result in Fig. 8 supports our argument. In this scenario, the flow caching achieves higher throughput than without caching even for a flow length as short as 100 packets.

4) Multi-core Scaling: As mentioned earlier, we take the multi-core architecture into account in our design. To explore how the hardware classification extension scales with the number of CPU cores we run a test with up to eight CPU cores in total (4 physical CPU cores with Hyper-Threading [17]). We create two receive queues for each CPU core, one for matched flows and the other for unmatched flows. We generate a load of 100 UDP flows at $5.5 \mathrm{M}$ pps. All flows match entries in the Flow Director filter. The test result in Fig. 9 shows how the hardware classification extension scales with an increasing number of CPU cores.

We make three interesting observations in the test results. First, the performance more than doubles when increasing the number of CPUs from one to two. This is likely due to the fact that our system is configured to have only the first CPU core handling all processes in the system. Thus, the first CPU has fewer cycles available for packet processing, and yields a lower throughput compared to other CPU cores. Second, the performance per CPU core degrades when HyperThreading is used (i.e., when using 5-8 CPU cores). According to [18], cache-friendly applications might suffer from HyperThreading because logical processors might be competing for access to the cache, something which might result in performance degradation. We believe that this can explain the performance degradation we experience since our system uses a cache-friendly queue mapping scheme. Finally, when HyperThreading is used, the forwarding throughput measurements show comparatively high variations. This is likely due to the randomness in resource contention. With Hyper-Threading, two logical CPU cores share the same physical CPU core and thus compete for resources. Since packets are arriving at random time to different CPU cores, we might get different levels of resource contention in different tests.

\section{CONClusions And Future Work}

In this paper, we propose an architecture, called hardware classification extension, to improve the lookup performance of PC-based OpenFlow with the use of on-board NIC hardware classification to offload the CPU from the lookup processing. Our solution is based on caching of flows and creating a

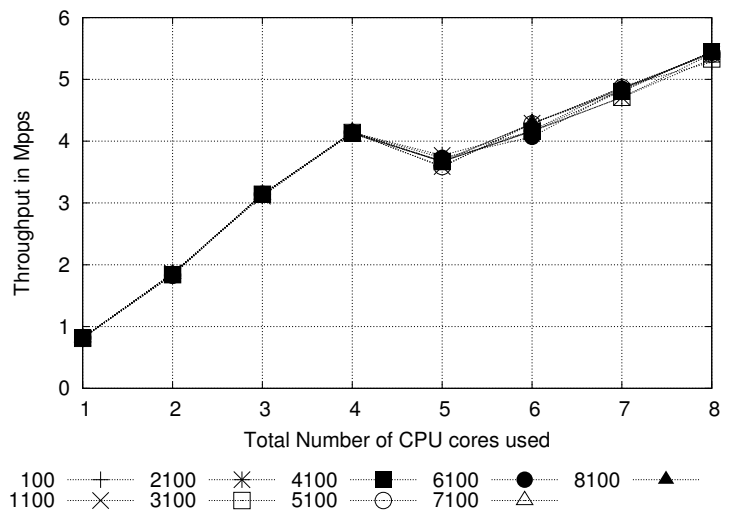

Fig. 9. Multi-core Scaling

fast path in the lookup process. Specifically, we use the Flow Director feature of a regular Intel NIC for caching flow entries in hardware. Compared to using FPGAs and NPs, our approach requires very modest changes in software and we use standard hardware support.

We outline a design architecture and present an experimental evaluation to investigate the performance gain we can achieve with the hardware classification extension as well as the overhead that it might incur incase of cache misses. This overhead is negligeble. More importantly, there is a certain cost associated with setting up a hardware cache entry, and this cost is amortized over the time during which the flow is active. We demonstrate how our solution significantly improves the throughput in a worst-case traffic scenario as well as in a more realistic scenario. In the worst-case scenario, a flow length of 10000 64-byte packets is needed, but in a more realistic setting our solution outperform the standard OpenFlow forwarding even for a flow length of 100 64-byte packets.

In our investigations, the Flow Director shows a promising potential for flow caching. We have found some limitations like limited support for wildcards, but we believe that these could be addressed by a more advanced version of the Flow Director. Alternatively, a solution based on TCAM (Ternary Content Addressable Memory) could be used instead.

Apart from the Flow Director feature, modern NICs have many other interesting features and capabilities that can enhance the hardware classification extension further. For example, the ability to assign traffic classes and the possibility to adjust packet buffers' size could be used to fine-tune the hardware classification extension. Further investigation on these parameters could lead to an improvement of our architecture.

In addition, the TYAN S7002 motherboard that we use can accommodate two physical processors and two memory nodes but we use only one physical processor and one memory node in our setup. Adding more physical CPU and memory node can improve our performance further. This motherboard has Non-Uniform Memory Access (NUMA) support, which means that there are different buses to different memory nodes and a processor can take longer time to access a memory node that 
is not on its local bus. Thus, more investigation on a suitable configuration that best utilizes NUMA architecture is needed.

When it comes to traffic characteristics, earlier studies [19], [20], [21] show that the majority of network traffic flows are short-lived flows. However, only a small number of minority (long-lived) flows are accounted for a high proportion of the total amount of traffic on a given link. This is usually described as mice and elephants, where mice represent small shortlive flows and elephants represent flows that account for the majority of the traffic on the link. Given this traffic pattern, it would make sense to selectively cache only the long-lived flows and ignore the short-lived flows. This idea of focusing on the elephants to make overall improvements in general has been proposed in several types of related studies [22], [23], [24], [25]. In addition, this choice of selective caching would make it possible to accommodate the relevant flows within the table size limits of the Flow Director (of maximum 8K entries). The results from our experiments are encouraging and we plan further studies on more advanced caching schemes in order to optimize our solution for actual network traffic characteristics.

\section{ACKNOWLEDGMENT}

We would like to thank Daniel Turull for his help in troubleshooting hardware problem and software bug, and Robert Olsson for valuable insights and discussions related to the Intel $8259910 \mathrm{GbE}$ controller NIC.

\section{REFERENCES}

[1] N. Chowdhury and R. Boutaba, "Network virtualization: state of the art and research challenges," Communications Magazine, IEEE, vol. 47, no. 7, pp. 20-26, jul. 2009 .

[2] P. Szegedi, S. Figuerola, M. Campanella, V. Maglaris, and C. CervelloPastor, "With evolution for revolution: managing federica for future internet research," Communications Magazine, IEEE, vol. 47, no. 7, pp. 34-39, jul. 2009.

[3] J. S. Turner, "A proposed architecture for the geni backbone platform," in ANCS '06: Proceedings of the 2006 ACM/IEEE symposium on Architecture for networking and communications systems. New York, NY, USA: ACM, 2006, pp. 1-10.

[4] H. Harai, "Designing new-generation network: Overview of akari architecture design," in Asia Communications and Photonics Conference and Exhibition. Optical Society of America, 2009, p. FL2. [Online]. Available: http://www.opticsinfobase.org/abstract.cfm?URI= ACP-2009-FL2

[5] K.-K. Yap, T.-Y. Huang, B. Dodson, M. S. Lam, and N. McKeown, "Towards software-friendly networks," in Proceedings of the first ACM asia-pacific workshop on Workshop on systems, ser. APSys ' 10. New York, NY, USA: ACM, 2010, pp. 49-54. [Online]. Available: http://doi.acm.org/10.1145/1851276.1851288

[6] N. McKeown, T. Anderson, H. Balakrishnan, G. Parulkar, L. Peterson, J. Rexford, S. Shenker, and J. Turner, "Openflow: enabling innovation in campus networks," SIGCOMM Comput. Commun. Rev., vol. 38, no. 2, pp. 69-74, 2008.

[7] The OpenFlow Consortium, "Openflow switching reference system," January 2011. [Online]. Available: http://www.openflowswitch.org

[8] A. Bianco, R. Birke, L. Giraudo, and M. Palacin, "Openflow switching: Data plane performance," in Communications (ICC), 2010 IEEE International Conference on, May 2010, pp. 1 -5.
[9] Intel, "Product brief: Intel 8259910 gigabit ethernet controller," 2009. [Online]. Available: http://edc.intel.com/Link.aspx?id=2376

[10] J. Naous, D. Erickson, G. A. Covington, G. Appenzeller, and N. McKeown, "Implementing an openflow switch on the netfpga platform," in ANCS '08: Proceedings of the 4th ACM/IEEE Symposium on Architectures for Networking and Communications Systems. New York, NY, USA: ACM, 2008, pp. 1-9.

[11] Y. Luo, P. Cascn, E. Murray, and J. O. Lopera, "Accelerating openflow switching with network processors," in ANCS '09: Proceedings of the 5th ACM/IEEE Symposium on Architectures for Networking and Communications Systems. New York, NY, USA: ACM, 2009.

[12] V. Tanyingyong, M. Hidell, and P. Sjödin, "Improving pc-based openflow switching performance," in Proceedings of the 6th ACM/IEEE Symposium on Architectures for Networking and Communications Systems, ser. ANCS '10. New York, NY, USA: ACM, 2010, pp. 13:113:2. [Online]. Available: http://doi.acm.org/10.1145/1872007.1872023

[13] S. Bradner and J. McQuaid, "Benchmarking Methodology for Network Interconnect Devices," Internet Engineering Task Force, RFC 2544, Mar. 1999. [Online]. Available: http://www.rfc-editor.org/rfc/rfc2544.txt

[14] R. Olsson, "pktgen the linux packet generator," in Linux Symposium, vol. 2, 2005, pp. 11-24. [Online]. Available: http://www.linuxsymposium.org/2005/linuxsymposium\_procv2.pdf

[15] D. Turull, "Open source traffic analyzer," Master's thesis, KTH Information and Communication Technology, 2010. [Online]. Available: http://tslab.ssvl.kth.se/pktgen/docs/DanielTurull-thesis.pdf

[16] "Bifrost project." [Online]. Available: http://bifrost.slu.se/

[17] D. T. Marr, F. Binns, D. L. Hill, G. Hinton, D. A. Koufaty, J. A. Miller, and M. Upton, "Hyper-threading technology architecture and microarchitecture," Intel Technology Journal, vol. 6, no. 1, pp. 4-15, 2002. [Online]. Available: http://developer.intel.com/technology/ itj/2002/volume06issue01/vol6iss1_hyper_threading_technology.pdf

[18] T. Leng, R. Ali, J. Hsieh, V. Mashayekhi, and R. Rooholamini, "An empirical study of hyper-threading in high performance computing clusters," in proceedings of The Third LCI International Conference on Linux Clusters: The HPC Revolution 2002, ser. Linux Clusters: The HPC Revolution, 2002. [Online]. Available: http://www.democritos.it/ activities/IT-MC/cluster_revolution_2002/PDF/11-Leng_T.pdf

[19] K. Thompson, G. Miller, and R. Wilder, "Wide-area internet traffic patterns and characteristics," Network, IEEE, vol. 11, no. 6, pp. $10-$ 23, nov/dec 1997.

[20] N. Brownlee and K. Claffy, "Understanding internet traffic streams: dragonflies and tortoises," Communications Magazine, IEEE, vol. 40, no. 10 , pp. 110 - 117, Oct. 2002.

[21] K. chan Lan and J. Heidemann, "A measurement study of correlations of internet flow characteristics," Computer Networks, vol. 50, no. 1, pp. 46 - 62, 2006. [Online]. Available: http://www.sciencedirect.com/science/ article/B6VRG-4G7X5D3-2/2/68cc7a078b8a785cb729980fe4155d79

[22] N. Brownlee, "Some observations of internet stream lifetimes," in Passive and active network measurement: 6th international workshop, PAM 2005, Boston, MA, USA, March 31-April 1, 2005 : proceedings, ser. Lecture notes in computer science. Springer, 2005, pp. 265-277. [Online]. Available: http://books.google.com/books?id=t60_xTVn15gC

[23] C. Estan and G. Varghese, "New directions in traffic measurement and accounting: Focusing on the elephants, ignoring the mice," ACM Trans. Comput. Syst., vol. 21, pp. 270-313, August 2003. [Online]. Available: http://doi.acm.org/10.1145/859716.859719

[24] J. Erman, A. Mahanti, and M. Arlitt, "Byte me: a case for byte accuracy in traffic classification," in Proceedings of the $3 r d$ annual ACM workshop on Mining network data, ser. MineNet '07. New York, NY, USA: ACM, 2007, pp. 35-38. [Online]. Available: http://doi.acm.org/10.1145/1269880.1269890

[25] J. Arkko, B. Briscoe, L. Eggert, A. Feldmann, and M. Handley, "Dagstuhl perspectives workshop on end-to-end protocols for the future internet," SIGCOMM Comput. Commun. Rev., vol. 39, pp. 42-47, March 2009. [Online]. Available: http://doi.acm.org/10.1145/1517480.1517489 